PROCEEDINGS OF THE

AMERICAN MATHEMATICAL SOCIETY

Volume 125, Number 4, April 1997, Pages 961-969

S 0002-9939(97)03619-8

\title{
TORSION-FREE DUALITY IS WARFIELD
}

\author{
T. FATICONI, H. P. GOETERS, C. VINSONHALER, AND W. J. WICKLESS
}

(Communicated by Wolmer V. Vasconcelos)

\begin{abstract}
We show that, under certain natural conditions, a duality discovered by R. B. Warfield, Jr., is the only duality on categories of finite-rank torsion-free modules over Dedekind domains.
\end{abstract}

In a seminal paper $[\mathrm{W}], \mathrm{R}$. B. Warfield extended the theory of vector space duality to certain categories of torsion-free groups of finite rank. His work has spawned a host of applications and generalizations. For examples, see [L2], [R], $[\mathrm{G}],[\mathrm{VW}]$. In particular, the work in [L2] and [R] extends Warfield duality to modules over Dedekind domains. The purpose of this paper is to show that under a few standard conditions, Warfield duality is the only duality on categories of torsion-free modules of finite rank over Dedekind domains (Theorem 9). Before making ourselves more precise, we establish some definitions and notation.

Throughout, $R$ will be a Dedekind domain with quotient field $Q$. We assume familiarity with the standard properties of such rings, as found in Chapter 10 of $[\mathrm{J}]$, for example. All of our $R$-modules are torsion-free of finite rank, unless otherwise indicated, and all homomorphisms are $R$-homomorphisms. Two rank-one modules $A$ and $B$ are quasi-isomorphic, $A \dot{\sim} B$, if there is an embedding $f: A \rightarrow B$ and a nonzero $r \in R$ such that $r B \subseteq f A$. The type of a rank-one module $A$ is the set of submodules of $Q$ that are quasi-isomorphic to $A$. The typeset of a module $G$, typeset $(G)$, is the set of types of rank-one pure submodules of $G$. (A submodule $A$ of a torsion-free module $B$ is pure if the quotient module $B / A$ is torsion-free.) We view typesets as subsets of the distributive lattice of all types. If $A$ and $B$ are submodules of $Q$, then type $(A) \leq \operatorname{type}(B)$ if and only if $\operatorname{Hom}(A, B) \neq 0$, in which case $\operatorname{End}(A) \subseteq \operatorname{End}(B)$ as subrings of $Q$. Also, type $(A) \vee \operatorname{type}(B)=\operatorname{type}(A+B)$ and type $(A) \wedge \operatorname{type}(B)=\operatorname{type}(A \cap B)$. For a module $G$, the inner type of $G, I T(G)$, is the infimum of typeset $(G)$. Dually, $O T(G)$, the outer type of $G$, is the supremum of the set of types of rank-one factors of $G$. As shown in [W], [L2] and [R], IT $(G)$ is the meet of a finite number of types in $\operatorname{typeset}(G)$, and $O T(G)$ is the join of a finite number of types of rank-one factors of $G$. If $X$ is a submodule of the quotient field $Q$, a module $G$ is called $X$-locally free if $O T(G) \leq$ type $X$ and $\{p$ a prime ideal of $R \mid p X=X\}=\{p \mid p G=G\}$. As is standard, we identify $\operatorname{End}(X)=\operatorname{End}_{R}(X)$ with the subring of $Q$ generated by $\left\{p^{-1} \mid p X=X\right\}$. Note that if $G$ is $X$-locally free, then the $R$-module structure on $G$ extends to an $\operatorname{End}(X)$-module structure.

Throughout, $\mathcal{C}$ and $\mathcal{D}$ are categories of torsion-free $R$-modules of finite rank with the usual module homomorphisms. We assume that $\mathcal{C}$ is closed under finite direct

Received by the editors March 23, 1995 and, in revised form, September 25, 1995.

1991 Mathematics Subject Classification. Primary 20K15, 20K40, 20 C05.

(C)1997 American Mathematical Society 
sums and pure rank-one submodules. We also fix an additive duality $F: \mathcal{C} \rightarrow \mathcal{D}$ that takes rank- $n$ modules to rank- $n$ modules ( $F$ preserves rank). As in $[\mathrm{AF}]$, a duality from $\mathcal{C}$ to $\mathcal{D}$ is a pair of contravariant functors $\left(F, F^{-1}\right)$ such that $F \cdot F^{-1}$ and $F^{-1} \cdot F$ are each naturally equivalent to the appropriate identity functor. It is easy to check that, in the presence of our other assumptions, $F$ preserves rank if and only if $F$ takes rank-one modules to rank-one modules.

Fix a subgroup $X$ of $Q$ and let $L F(X)$ be the category of $X$-locally free modules and module homomorphisms. Warfield, Lady and Reid showed that the functor $\operatorname{Hom}(-, X)$ is a duality from $L F(X)$ into itself. We refer to a duality of this form as Warfield duality. The category $L F(X)$ is closed under direct sums and pure rank-one submodules, and Warfield duality preserves rank. These assumptions on our category $\mathcal{C}$ and functor $F$ are necessary and minimal in the sense that if any one is omitted, then $F$ need not be Warfield. Examples at the end of the paper illustrate this point.

The typeset of the category $\mathcal{C}$ is the set of types of all rank-one modules in $\mathcal{C}$. By our assumptions on $\mathcal{C}$, typeset $(\mathcal{C})$ is the union of the typesets of modules in $\mathcal{C}$.

Lemma 1. (a) If $\tau, \sigma \in \operatorname{typeset}(\mathcal{C})$, then $\tau \wedge \sigma \in \operatorname{typeset}(\mathcal{C})$.

(b) If $G \in \mathcal{C}$, then $\operatorname{IT}(G) \in \operatorname{typeset}(\mathcal{C})$.

Proof. (a) Let $A$ and $B$ be rank-one modules in $\mathcal{C}$ such that $\tau=\operatorname{type}(A)$ and $\sigma=\operatorname{type}(B)$. There are injections $\varphi_{1}: A \rightarrow Q$ and $\varphi_{2}: B \rightarrow Q$. Let $\varphi: A \oplus B \rightarrow Q$ be the module homomorphism defined by $\varphi(a, b)=\varphi_{1}(a)-\varphi_{2}(b)$. Then $\operatorname{ker} \varphi$ is a pure rank-one submodule of $A \oplus B \in \mathcal{C}$ that is isomorphic to $\varphi_{1}(A) \cap \varphi_{2}(B)$. Since $\mathcal{C}$ is closed under pure rank-one submodules, $\operatorname{ker} \varphi$ is a rank-one module in $\mathcal{C}$ of type $\sigma \wedge \tau$.

(b) As noted above, the inner type of a module $G$ in $\mathcal{C}$ is the meet of a finite number of types in typeset $(G)$. Hence (b) follows directly from (a).

If $A$ is a rank-one module in $\mathcal{C}$, the endomorphism ring of $A$ can be naturally identified with a subring of $Q: \operatorname{End}(A)=\{q \in Q: q A \subseteq A\}$. With this identification, we have

Lemma 2. There are subrings $S$ and $T$ of $Q$, each containing $R$, such that for all rank-one $A \in \mathcal{C}$, we have $\operatorname{End}(A)=S$ and $\operatorname{End}(F A)=T$. Moreover, the functor $F$ induces a ring isomorphism $\rho: S \rightarrow T$.

Proof. Assume first that $A$ and $B$ are rank-one modules in $\mathcal{C}$ with type $(A) \leq$ type $(B)$. This type relationship implies $\operatorname{End}(A) \subseteq \operatorname{End}(B)$ so that $\operatorname{End}(F A) \subseteq$ $\operatorname{End}(F B)$. Since $\operatorname{Hom}(A, B) \neq 0$, we obtain $\operatorname{Hom}(F B, F A) \neq 0$. Because $F A, F \bar{B}$ are rank-one, $\operatorname{End}(F B) \subseteq \operatorname{End}(F A)$ so that $\operatorname{End}(F A)=\operatorname{End}(F B)$. By symmetry, $\operatorname{End}(A)=\operatorname{End}(B)$. To handle the case where $A$ and $B$ have incomparable types, we can use Lemma 1 to find a rank-one module $C$ in $\mathcal{C}$ with $\operatorname{type}(C)=\operatorname{type}(A) \wedge$ $\operatorname{type}(B)$, and argue as above to get $\operatorname{End}(A)=\operatorname{End}(C)=\operatorname{End}(B)$. Finally, the additive functor $F$ induces an isomorphism $\rho: \operatorname{End}(A) \rightarrow \operatorname{End}(F A)$.

We call $F$ normalized if the induced isomorphism $\rho$ of Lemma 2 is the identity. In general, using Lemma 2, the objects in $\mathcal{C}$ may be regarded canonically as $S$ modules, since $\mathcal{C}$ is closed under rank-one pure submodules. Define a new category $\mathcal{C}_{T}$ as follows. The objects in $\mathcal{C}_{T}$ are the objects of $\mathcal{C}$ regarded as $T$-modules under the scalar multiplication $t \cdot m=\rho^{-1}(t) m$. The morphisms of $\mathcal{C}_{T}$ are then the $T$ homomorphisms. Note that these are precisely the $S$-homomorphisms in $\mathcal{C}$ (which 
coincide with the $R$-homomorphisms). It follows that the identity provides a functor $\mathcal{C}_{T} \rightarrow \mathcal{C}$, which we also denote by $\rho$. We call the composition $F_{\nu}=(F \cdot \rho): \mathcal{C}_{T} \rightarrow \mathcal{D}$ the normalization of the functor $F$. The following facts are routine: For each rank-one module $A$ in $\mathcal{C}_{T}, \operatorname{End}(A)=T$ and the isomorphism $\operatorname{End}(A) \simeq \operatorname{End}\left(F_{\nu} A\right)$ induced by $F_{\nu}$ is the identity. In particular, $F_{\nu}$ is normalized.

Lemma 3. Let $F: \mathcal{C} \rightarrow \mathcal{D}$ be normalized. Then for any rank-one $A, B \in \mathcal{C}$, $\operatorname{Hom}(A, B) \simeq \operatorname{Hom}(F B, F A)$ as $R$-modules.

Proof. It follows directly from our definitions that $\operatorname{Hom}(A, B) \simeq \operatorname{Hom}(F B, F A)$ as $T$-modules, where $R \subseteq T=\operatorname{End}(F A)$.

Distinct from the meet and join operations, there are standard notions of addition and subtraction on the lattice of types. These may be defined as follows: If $A$ and $B$ are submodules of $Q$ of types $\tau$ and $\sigma$, respectively, then $\tau+\sigma$ is the type of the product $A B \subseteq Q$. If $\tau \leq \sigma$, then $\sigma-\tau$ is the type of the conductor $(B: A)=\{q \in Q: q A \subseteq B\}$.

Lemma 4. Assume $F: \mathcal{C} \rightarrow \mathcal{D}$ is normalized. For any rank-one modules $A, B \in \mathcal{C}$ and $D \in \mathcal{D}$,

$$
\operatorname{type}(A)+\operatorname{type}(F A)=\operatorname{type}(B)+\operatorname{type}(F B)=\operatorname{type}(D)+\operatorname{type}\left(F^{-1} D\right) .
$$

Proof. First assume type $(A) \leq \operatorname{type}(B)$. Then $\operatorname{Hom}(A, B)$ has rank one and, since $F$ is a normalized duality, $\operatorname{Hom}(A, B) \simeq \operatorname{Hom}(F B, F A)$ as $R$-modules by Lemma 3 . By the usual equations for types and because $F$ preserves rank,

$$
\begin{aligned}
\operatorname{type}(B)-\operatorname{type}(A) & =\operatorname{type} \operatorname{Hom}(A, B)=\operatorname{type} \operatorname{Hom}(F B, F A) \\
& =\operatorname{type}(F A)-\operatorname{type}(F B) .
\end{aligned}
$$

Thus, $\operatorname{type}(A)+\operatorname{type}(F A)=\operatorname{type}(B)+\operatorname{type}(F B)$.

In general, given rank-one modules $A$ and $B$ in $\mathcal{C}$, there is, by Lemma 1, a rankone module $C$ in $\mathcal{C}$ such that type $(C)=\operatorname{type}(A) \wedge$ type $(B)$. Then by the previous paragraph,

$$
\operatorname{type}(A)+\operatorname{type}(F A)=\operatorname{type}(C)+\operatorname{type}(F C)=\operatorname{type}(B)+\operatorname{type}(F B) .
$$

Finally, because each rank-one $D \in \mathcal{D}$ satisfies $F C \simeq D$ for some rank-one $C \in \mathcal{C}$, we have

$$
\operatorname{type}(C)+\operatorname{type}(F C)=\operatorname{type}\left(F^{-1} D\right)+\operatorname{type}(D) .
$$

Lemma 5. Let $A, B, C, D$ be rank-one $R$-modules that are all divisible by the same primes in $R$, and assume $f: A \rightarrow B$ is an embedding. Then the R-modules $\operatorname{Hom}(A, B)$ and $\operatorname{Hom}(C, D)$ are isomorphic if and only if there is an embedding $g: C \rightarrow D$ such that $B / f A \simeq D / g C$.

Proof. Since $f A$ and $B$ are rank-one modules over a Dedekind domain, it follows that $\operatorname{Hom}(f A, B)_{p}$ can be identified with $\operatorname{Hom}\left(f A_{p}, B_{p}\right)$ and $f A_{p}=p^{k} B_{p}$ for some $k \geq 0$. Moreover, if $p B \neq B$, then $k$ is precisely the $p$-height of $f$ in $\operatorname{Hom}(A, B)$ $\left(f \in p^{k} \operatorname{Hom}(A, B) \backslash p^{k+1} \operatorname{Hom}(A, B)\right)$.

Suppose $\theta: \operatorname{Hom}(A, B) \rightarrow \operatorname{Hom}(C, D)$ is an isomorphism. Because the embedding $g=\theta f$ has the same $p$-height as $f$, it follows that $g C_{p}=p^{k} D_{p}$. That is, $(B / f A)_{p} \simeq B_{p} / f A_{p} \simeq D_{p} / g C_{p} \simeq(D / g C)_{p}$. We may conclude that $B / f A \simeq$ $D / g C$. 
Conversely, if $g: C \rightarrow D$ is an embedding such that $B / f A \simeq D / g C$, then for each prime $p$, the $p$-height of $f$ in $\operatorname{Hom}(A, B)$ equals the $p$-height of $g$ in $\operatorname{Hom}(C, D)$. Thus, the mapping defined by $f \rightarrow g$ provides an isomorphism from $\operatorname{Hom}(A, B)$ to $\operatorname{Hom}(C, D)$.

Continuing with the normalized hypothesis, let $A_{0}$ be a fixed rank-one module in $\mathcal{C}$. Using Lemma 5, we can construct a rank-one module $X \simeq A_{0} \otimes_{R} F A_{0}$ such that $\operatorname{Hom}\left(F A_{0}, X\right) \simeq \operatorname{Hom}\left(R, A_{0}\right) \simeq A_{0}$. Plainly,

$$
\operatorname{type}(X)=\operatorname{type}\left(A_{0}\right)+\operatorname{type}\left(F A_{0}\right) \text {. }
$$

By Lemma 4,

$$
\operatorname{type}(X)=\operatorname{type}(A)+\operatorname{type}(F A)=\operatorname{type}(D)+\operatorname{type}\left(F^{-1} D\right)
$$

for all rank-one $A \in \mathcal{C}$ and $D \in \mathcal{D}$. For this $X$, we denote $W=\operatorname{Hom}(-, X)$. As indicated by the next lemma, the use of rank-one modules is central in our arguments.

Lemma 6. Let $F: \mathcal{C} \rightarrow \mathcal{D}$ be normalized and let $A$ be a rank-one module in $\mathcal{C}$. Then as $R$-modules, $A \dot{\sim} W F(A)$ and $W A \dot{\sim} F A$. Similarly, if $D$ is rank-one in $\mathcal{D}$, then $D \dot{\simeq} W F^{-1} D$ and $W D \dot{\sim} F^{-1} D$.

Proof. By $(\dagger)$, type $(A) \leq \operatorname{type}(X)$. Then, using $(\dagger)$ again,

$$
\operatorname{type}(F A)=\operatorname{type}(X)-\operatorname{type}(A)=\operatorname{type} \operatorname{Hom}(A, X)=\operatorname{type}(W A) \text {. }
$$

Since $F A$ and $W A$ are rank-one, $F A \dot{\simeq} W A$. Clearly, $W A$ and thus $F A$ are modules over $\operatorname{End}(X)$, a subring of $Q$. Since $F$ is normalized, an application of Lemma 3 shows that $A$ is also a module over $\operatorname{End}(X)$. Thus, $A$ is $X$-locally free and $W^{2} A \simeq A$ by Warfield duality. It follows that $W F A \dot{\simeq} A$. A similar argument shows $D \dot{\simeq} W F^{-1} D$ and $W D \dot{\simeq} F^{-1} D$.

Proposition 7. The category $\mathcal{D}$ is a full subcategory of $L F(X)$.

Proof. We assume, without loss of generality, that $F: \mathcal{C} \rightarrow \mathcal{D}$ is normalized. Choose $H \in \mathcal{D}$. Since $F$ is a duality, there exists $G \in \mathcal{C}$ with $F G \simeq H$. We first show $O T(H) \leq \operatorname{type}(X)$. Let $C_{1}, \ldots, C_{n}$ be a maximal independent set of pure rankone submodules of $G$. By Lemma 1 , there is a rank-one module $C_{0} \in \mathcal{C}$ with type $\left(C_{0}\right)=\bigwedge$ type $\left(C_{i}\right)$. Fix embeddings $C_{0} \rightarrow C_{i}$. The composite maps $\varepsilon_{i}:$ $C_{0} \rightarrow C_{i} \rightarrow G$ are morphisms in $\mathcal{C}$. Moreover, because $F$ is a normalized duality, it is easy to check that the dual maps $F \varepsilon_{i}: F G \rightarrow F C_{0}$ provide a maximal $R$ independent subset of the $R$-module $\operatorname{Hom}\left(F G, F C_{0}\right)$ of cardinality equal to the $R$-rank of $G$. Since $\operatorname{rank}(F G)=\operatorname{rank}(G)$, Proposition 2.5 of $[\mathrm{R}]$ guarantees that $O T(H)=O T(F G) \leq \sup \left\{\operatorname{type}\left(F C_{i}\right)\right\} \leq \operatorname{type}(X)$, the last inequality by $(\dagger)$.

Denote by $E$ the endomorphism ring of $X$, regarded as a subring of $Q$. To complete that proof that $H$ is $X$-locally free, we show that $H$ is an $E$-module. As noted in the proof of Lemma 6, each rank-one module in $\mathcal{C}$ is an $E$-module. Since $\mathcal{C}$ is closed under rank-one pure submodules, each module in $\mathcal{C}$ is an $E$-module. Since $F$ is normalized, each module in $\mathcal{D}$ is an $E$-module.

Our goal is to prove that $F$ (normalized) and $W$ are equivalent. To make this more precise, let $i_{\mathcal{D}}: \mathcal{D} \rightarrow L F(X)$ be the inclusion functor guaranteed by Proposition 7 . We prove that $\mathcal{C}$ is also a full subcategory of $L F(X)$ and that $W i_{\mathcal{D}} F$ is equivalent to $i_{\mathcal{C}}$, the embedding of $\mathcal{C}$ in $L F(X)$. We do this first for rank-one modules. 
Proposition 8. Let $F: \mathcal{C} \rightarrow \mathcal{D}$ be normalized. On finite direct sums of rankone modules in $\mathcal{C}$, the restriction of the functor $W i_{\mathcal{D}} F$ is naturally equivalent to inclusion in $\operatorname{LF}(X)$.

Proof. Note that finite direct sums of rank-one modules in $\mathcal{C}$ form a full subcategory of $L F(X)$ by the proof of Lemma 6 . Since all the functors in the theorem are additive, it suffices to establish the theorem for rank-one modules in $\mathcal{C}$. Let $j=$ $W i_{\mathcal{D}} F$ and let $A_{0}$ be the rank-one module used to construct $X$ in the paragraph following Lemma 5. By construction, $A_{0} \cong j A_{0}$, so we let $\theta_{0}: A_{0} \rightarrow j A_{0}$ be a fixed module isomorphism. Suppose $B$ is a rank-one module in $\mathcal{C}$ with type $B \leq$ type $A_{0}$ and let $\varphi$ be any nonzero homomorphism from $B$ to $A_{0}$. The covariant functor $j$ induces an $R$-module isomorphism from $\operatorname{Hom}\left(B, A_{0}\right)$ to $\operatorname{Hom}\left(j B, j A_{0}\right)$. Moreover, by Lemma 6 , the rank-one modules $A_{0}, B, j A_{0}, j B$ are all divisible by the same primes, namely $\{p$ prime $\mid p X=X\}$. By Lemma $5, A_{0} / \varphi B \simeq j A_{0} / j \varphi(j B)$. Since $\theta_{0}: A_{0} \simeq j A_{0}$ is an isomorphism, $A_{0} / \varphi B \simeq j A_{0} / \theta_{0} \varphi B$, so that $j A_{0} / j \varphi(j B) \simeq$ $j A_{0} / \theta_{0} \varphi B$. Because $j A_{0}$ has rank one, the submodules $j \varphi(j B)$ and $\theta_{0} \varphi B$ must be equal. The confluence of these remarks implies that the map $(j \varphi)^{-1} \theta_{0} \varphi$ (regarded as a map from $B$ into the divisible hull of $j B$ ) carries $B$ into $j B$. Thus $\theta_{B}=(j \varphi)^{-1} \theta_{0} \varphi$ is the unique isomorphism from $B$ to $j B$ completing the following commutative diagram:

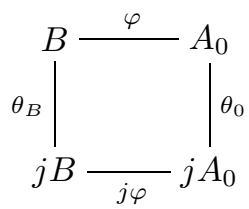

Any nonzero $\varphi^{\prime}: B \rightarrow A_{0}$ is dependent on $\varphi$; that is, $r \varphi^{\prime}=s \varphi$ for some nonzero $r, s \in R$. Using this fact, it is easy to check that $(j \varphi)^{-1} \theta_{0} \varphi=\left(j \varphi^{\prime}\right)^{-1} \theta_{0} \varphi^{\prime}$ so that $\theta_{B}$ is independent of the choice of the nonzero embedding $\varphi$, given the initial choice of $\theta_{0}$.

A similar argument provides an isomorphism $\theta_{B}: B \rightarrow j B$ for a rank-one module $B$ in $\mathcal{C}$ with type $B \geq$ type $A_{0}$. If $B$ is a rank-one module in $\mathcal{C}$ with type $B$ and type $A_{0}$ incomparable, then, by Lemma 1 , there exists a rank-one module $B^{\prime}$ in $\mathcal{C}$ with type $B^{\prime}=$ type $B \wedge$ type $A_{0}$. By the previous paragraph, the map $\theta_{0}$ uniquely determines an isomorphism $\theta_{B^{\prime}}: B^{\prime} \rightarrow j B^{\prime}$. Let $\varphi: B^{\prime} \rightarrow B$ be a nonzero embedding. Then we can construct a unique isomorphism $\theta_{B}$ making the diagram

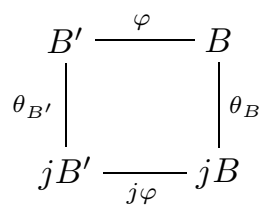

commute. As before, $\theta_{B}$ is independent of the choice of $\varphi$. The natural transformation $\theta$ as constructed provides an equivalence between $j=W i_{\mathcal{D}} F$ and inclusion into $L F(X)$ on direct sums of rank-one modules in $\mathcal{C}$. The routine details are omitted.

Relying heavily on Proposition 8, we extend the equivalence $\theta$ to all of $\mathcal{C}$.

Theorem 9. Let $\mathcal{C}$ and $\mathcal{D}$ be full subcategories of the category of torsion-free modules of finite rank and homomorphisms over a Dedekind domain $R$, with $\mathcal{C}$ closed 
under pure rank-one submodules and finite direct sums. If $F: \mathcal{C} \rightarrow \mathcal{D}$ is a normalized additive duality that preserves rank, then $F$ is naturally equivalent to Warfield duality.

Proof. As above, let $i_{\mathcal{D}}$ be the embedding of $\mathcal{D}$ as a full subcategory of the $X$ locally free modules, where $X$ is a submodule of $Q$ with $\operatorname{Hom}(F A, X) \simeq A$ and $\operatorname{type}(X)=\operatorname{type}(A)+\operatorname{type}(F A)$ for any $A$ in $\mathcal{C}$ with $\operatorname{rank}(A)=1$. We show $j=W i_{\mathcal{D}} F \sim i_{\mathcal{C}}$, where $W=\operatorname{Hom}(-, X)$ and $\iota_{C}$ is the inclusion of $\mathcal{C}$ in $L F(X)$. In particular, our discussion will show that any module in $\mathcal{C}$ is $X$-locally free. By Proposition 8 , the equivalence $j \sim i_{\mathcal{C}}$ holds (with a slight abuse of notation) if we restrict to direct sums of rank-one modules in $\mathcal{C}$.

Let $G$ be any element of $\mathcal{C}$. By Lemma 1 , there is a rank-one module $A \in \mathcal{C}$ with type $(A)=I T(G)$. If $\operatorname{rank}(G)=n$, let $B=A^{n}$ and construct an embedding $\varepsilon: B \rightarrow G$ in $\mathcal{C}$. Our first goal is to construct a map $\theta_{G}$ so that the following diagram commutes:

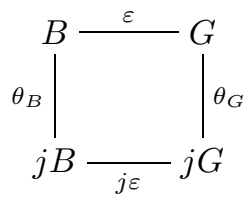

where $\theta_{B}$ is the isomorphism from Proposition 8 that gives the equivalence $W i_{\mathcal{D}} F \sim$ $i_{\mathcal{C}}$ on direct sums of rank-one modules in $\mathcal{C}$.

When extended to divisible hulls, the maps $\theta, \varepsilon$ and $j \varepsilon$ are all isomorphisms. Thus, there is a unique monomorphism $\theta_{G}: G \rightarrow Q j G$ that makes the following diagram commute:

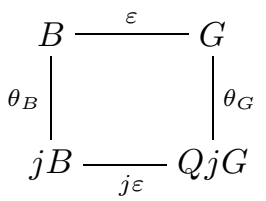

We show that $\theta_{G}$ actually maps $G$ isomorphically onto $j G$. Our first step is to show that for any rank-one submodule $H$ of $G$, we have $\theta_{G}(H) \subseteq j G$.

If $H$ is a pure rank-one submodule of $G$, then $\varepsilon^{-1} H=\{x \in B: \varepsilon x \in H\}$ is a pure rank-one submodule of $B$, whence an element of $\mathcal{C}$. Let $\iota: \varepsilon^{-1} H \rightarrow B$ be inclusion. By Proposition 8, the left square of the following is a commutative diagram; the right square is the commutative diagram above:

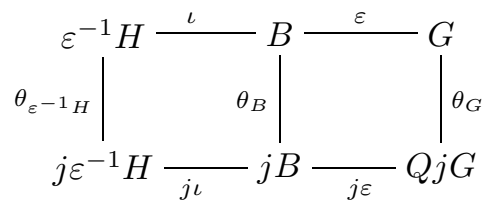

In the next diagram (below) the left square commutes, again by Proposition 8 ( $\bar{\varepsilon}$ is the restriction of $\varepsilon ; \iota_{H}$ is inclusion). Note that the composition $\varepsilon \iota$ equals $\iota_{H} \bar{\varepsilon}$, so that $j \varepsilon j \iota=j(\varepsilon \iota)=j\left(\iota_{H} \bar{\varepsilon}\right)=j \iota_{H} j \bar{\varepsilon}$. It follows that the outside square of the diagram below is the outside square of the previous diagram, whence is 
commutative:

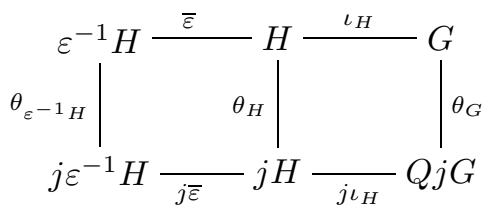

Since $\bar{\varepsilon}$ is a monomorphism, we can chase the diagram to show that the right square commutes. But $j \iota_{H}$ maps $j H$ into $j G$. It follows that $\theta_{G}(H) \subseteq j G$, as desired.

Our next step is to show that $\theta_{G}(H)$ is a pure submodule of $j G$. As the above diagram shows, this is equivalent to proving that $j \iota_{H}$ is a pure embedding. First observe that $j$ provides an isomorphism from $\operatorname{End}(H)$ to $\operatorname{End}(j H)$. In particular, $p$ is a prime ideal in $\operatorname{End}(H)$, if and only if $j p$ is prime in $\operatorname{End}(j H)$. The following chain of equivalences is then easy to construct:

$\iota_{H}$ is a pure embedding

$\Leftrightarrow \iota_{H} \notin \operatorname{Hom}(H, G) p$ for each prime ideal $p$ in $\operatorname{End}(H)$

$\Leftrightarrow j \iota_{H} \notin \operatorname{Hom}(j H, j G) j p$ for each prime ideal $j p$ in $\operatorname{End}(j H)$

$\Leftrightarrow j \iota_{H}$ is a pure embedding.

Since $H$ was chosen pure in $G$, we conclude that $\theta_{G}(H)$ is pure in $j G$.

The final step is to show $\theta_{G}$ is onto. For this, it suffices to show that every pure rank-one submodule $K$ of $j G$ is in the image of $\theta_{G}$. By our arguments above, the rank-one pure submodule generated by $\varepsilon \theta_{B}^{-1}(j \varepsilon)^{-1} K$ will be carried into, whence onto, $K$ by $\theta_{G}$. This completes the proof that $\theta_{G}: G \rightarrow j G$ is an isomorphism. Note that since $j G=\operatorname{Hom}(F G, X)$ is $X$-locally free, then so is $G$.

Finally, assume we have constructed in this way an isomorphism $\theta_{G}: G \rightarrow j G$ for each $G$ in $\mathcal{C}$. It remains to show that the following diagram commutes for any homomorphism $\varphi: G \rightarrow K$ in $\mathcal{C}$ :

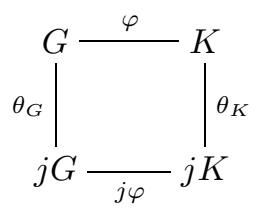

However, the diagram commutes if we restrict to any pure rank-one submodule of $G$, by Proposition 8 and the construction of the $\theta$ 's. This implies commutativity for the whole diagram and the proof is complete.

If $R=Z$ is the ring of integers, then the isomorphism classes of subrings of the rational numbers $Q$ each contain precisely one element. Moreover, if $S$ is a subring of $Q$, then the only automorphism of $S$ is the identity. It follows that in the case $R=Z$, every duality $F$ is normalized (see Lemma 2) and Theorem 9 takes a simpler form.

Corollary 10. Let $\mathcal{C}$ and $\mathcal{D}$ be full subcategories of the category of torsion-free abelian groups of finite rank and homomorphisms, with $\mathcal{C}$ closed under pure rankone subgroups and finite direct sums. If $F: \mathcal{C} \rightarrow \mathcal{D}$ is an additive duality that preserves rank, then $F$ is naturally equivalent to Warfield duality.

We conclude with some examples that justify the hypotheses of Theorem 9. Each example shows that the theorem fails when the corresponding hypothesis in 
the following list is omitted. The reader can easily check that the remaining three hypotheses are satisfied in each case.

(a) $\mathcal{C}$ is closed under pure rank-one submodules;

(b) $\mathcal{C}$ is closed under finite direct sums;

(c) $F$ is rank-preserving;

(d) $F$ is normalized.

For simplicity, we use $R=Z$ in the first three examples.

Example (a). Let $p_{1}, \ldots, p_{6}$ be distinct primes and for $1 \leq i \leq 6$ let $A_{i}$ be the subring of $Q$ generated by $p_{i}^{-1}$. Define

$$
G=\left[A_{1} \oplus A_{2} \oplus A_{3}\right] /\langle(1,1,1)\rangle
$$

and

$$
H=\left[A_{4} \oplus A_{5} \oplus A_{6}\right] /\langle(1,1,1)\rangle .
$$

Then $G$ and $H$ are rank-two abelian groups with $\operatorname{End}(G)=\operatorname{End}(H)=Z$ and $\operatorname{Hom}(G, X)$ not isomorphic to $H$ for any rank-one group $X$. Let $\mathcal{C}$ be the category of finite direct sums $G \oplus G \oplus \cdots \oplus G$ and $\mathcal{D}$ the category of finite direct sums $H \oplus H \oplus \cdots \oplus H$. The assignment $G \rightarrow H$ provides an additive duality from $\mathcal{C}$ to $\mathcal{D}$ that is not Warfield duality.

Example (b). Let $U$ and $V$ be disjoint infinite sets of rational primes. Define subgroups of $Q: A=\left\langle p^{-1} \mid p \in U\right\rangle, X=\left\langle p^{-3} \mid p \in U\right\rangle, B=\left\langle p^{-1} \mid p \in V\right\rangle, Y=$ $\left\langle p^{-3} \mid p \in V\right\rangle$. Then $A \subseteq X, B \subseteq Y$ satisfy the following properties: all are $p$-reduced for each prime $p$; type $(A)$ and type $(B)$ are incomparable; type $(X)$ and type $(Y)$ are incomparable; type $\operatorname{Hom}(A, X)$ and type $\operatorname{Hom}(B, Y)$ are incomparable. Let $\mathcal{C}=$ $\{A, B\}$ and $\mathcal{D}=\{\operatorname{Hom}(A, X), \operatorname{Hom}(B, Y)\}$. The assignment $A \rightarrow \operatorname{Hom}(A, X), B \rightarrow$ $\operatorname{Hom}(B, Y)$ gives a duality that is not Warfield.

Example (c). Let $G$ be a rank-two group with $\operatorname{End}(G)=Z$. Let $\mathcal{C}$ be the category of finite-rank free groups and $\mathcal{D}$ the category of groups $C \otimes G$, where $C \in \mathcal{C}$. The assignment $C \rightarrow \operatorname{Hom}(C, Z) \otimes G$ provides a duality that is not Warfield. Standard homological arguments show that $\operatorname{Hom}(-, G)$ is an inverse functor.

Example (d). Let $R=Z[i]$, the ring of integers in the algebraic number field obtained by adjoining $i=\sqrt{ }-1$ to the rationals. The ideal $3 R$ factors as a product of the prime ideals $p=(1+2 i) R$ and $q=(1-2 i) R$. Let $S=R_{p}$ and $T=R_{q}$ be the localization of the Dedekind domain $R$ at these primes. Complex conjugation provides a ring isomorphism $\rho: S \rightarrow T$. Let $\mathcal{C}$ be the category of all finite-rank free $S$-submodules. Each module $M$ in $\mathcal{C}$ is an abelian group that can be regarded as a $T$-module under the multiplication $t \cdot m=\rho^{-1}(t) m$. Let $\mathcal{D}$ be the category of these (free) $T$-modules, with $T$-module homomorphisms. It is easy to see that $\mathcal{D}$ coincides with the category of all finite-rank free $T$-modules; and that the identity $I$ provides a category equivalence from $\mathcal{C}$ to $\mathcal{D}$. The functor $W=\operatorname{Hom}(-, T)$ is a duality on $\mathcal{D}$, and the composition $F=W \cdot I$ is a duality from $\mathcal{C}$ to $\mathcal{D}$ that is not Warfield. Indeed, $\mathcal{C}$ and $\mathcal{D}$ are not subcategories of $L F(X)$ for any rank-one $R$-module $X$.

\section{ACKNowledgement}

This work grew out of a Workshop at the University of Connecticut that was sponsored in part by the UConn Research Foundation. We are grateful for their support. We are also indebted to the referee for a number of helpful suggestions. 


\section{REFERENCES}

[AF] F. W. Anderson and K. R. Fuller, Rings and categories of modules, Springer-Verlag, GTM 13 (1974), Springer-Verlag, New York. MR 54:5281

[G] H. P. Goeters, Warfield duality and extensions of modules over an integral domain, preprint.

[J] N. Jacobson, Basic Algebra II, W. H. Freeman, San Francisco (1983).

[L1] E. L. Lady, A seminar on splitting rings for torsion free modules over Dedekind domains, Lecture Notes in Mathematics 1006 (1982), Springer-Verlag, New York, 1-48. MR 85f: 13007

[L2] W W Warfield duality and rank one quasi-summands of tensor products of finite rank locally free modules over Dedekind domains, J. Algebra 121 (1989), 129-138. MR 90k:13007

[R] J. D. Reid, Warfield duality and irreducible groups, Cont. Math. 130 (1992), American Math. Society, Providence, 361-370. MR 93j:20114

[VW] C. Vinsonhaler and W. J. Wickless, Dualities for torsion-free abelian groups of finite rank, J. Algebra 128 (1990), 474-487. MR 91b:20076

[W] R. B. Warfield, Jr., Homomorphisms and duality for abelian groups, Math. Z. 107 (1968), 189-212. MR 38:5923

(T. Faticoni) Department of Mathematics, Fordham University, Bronx, New York 10458

(H. P. Goeters) Department of Mathematics, Auburn University, Auburn, Alabama 36849

(C. Vinsonhaler and W. J. Wickless) Department of Mathematics, University of ConNeCTiCut, Storrs, Connecticut 06269

E-mail address: vinson@uconnvm.uconn.edu

E-mail address: wjwick@math.uconn.edu 